\title{
PROPUESTA PARA UN PLAN INTEGRAL DE COMUNICACIÓN EN LA GESTIÓN DEPORTIVA
}

\section{PROPOSAL FOR A COMPREHENSIVE COMMUNICATION PLAN IN SPORTS MANAGEMENT}

Recibido el 7 de junio de 2021 / Aceptado el 3 de diciembre de 2021 / DOI: 10.24310/riccafd.2021.v10i3.12847 Correspondencia: Carlos A. Ballesteros-Herencia. cballesteros@hmca.uva.es

Ballesteros-Herencia, C.A. ${ }^{A-E}$

Facultad de Filosofía y Letras, Universidad de Valladolid, España.

Responsabilidades

${ }^{A}$ Diseño de la investigación, ${ }^{B}$ Recolector de datos, ${ }^{C}$ Redactor del trabajo, ${ }^{D}$ Tratamiento estadístico, ${ }^{E}$ Apoyo económico, Fldea original y coordinador de toda la investigación.

\section{RESUMEN}

El mundo del deporte ha aplicado las nuevas tecnologías desde su misma aparición, lo que ha conllevado un proceso continuo de cambio, modernización y profesionalización. En este contexto, la comunicación se ha convertido en un factor clave para la gestión eficiente de las organizaciones deportivas. Como resultado de una revisión sistemática de la literatura en castellano, se destacó cómo conseguir los objetivos de la entidad deportiva demanda actualmente una planificación integral de todo el proceso de la comunicación, con especial atención tanto a la comunicación interna como a la comunicación de retorno o interacción con los públicos propios de la organización deportiva.

\section{PALABRAS CLAVE}

comunicación deportiva, gestión deportiva, plan de comunicación.

\section{ABSTRACT}

The world of sport has applied new technologies since its very appearance, which has led to a profound process of change, modernization and professionalization. In this context, communication has become a key 
factor for the efficient management of sports organizations. As a result of the systematic review of the literature in Spanish, it was highlighted how achieving the objectives of the sports entity requires nowadays a comprehensive planning of the entire communication process, with special attention to both internal communication and communication of return or interaction. with the public of the sports organization.

\section{KEY WORDS}

sports communication, sports management, communication plan.

\section{INTRODUCCIÓN}

El deporte es un fenómeno social y cultural que abarca a muy diversos ámbitos. Superando su origen como actividad física se ha convertido tanto en una industria económica de primera magnitud como en un referente cultural y emocional que contribuye a definir identidades personales y nacionales. Así, son muy variadas las organizaciones públicas y privadas que prestan servicios en todos estos ámbitos. La gestión deportiva trata de generar una dinámica eficaz de los recursos humanos, materiales y económicos, utilizando a la comunicación como una herramienta crucial. Sin embargo, el estudio de la simbiosis entre comunicación y deporte ha sufrido un retraso, debido a la necesidad previa tanto de las Ciencias del Deporte como de las Ciencias de la Comunicación de "lograr legitimidad científica y un espacio institucional propio" (1).

Diversos estudios $(2,3,4)$ incluyen la comunicación de las instituciones deportivas como una de las cinco dimensiones básicas de actuación, a la altura de otras como recursos humanos, rendimiento de los deportistas, finanzas o calidad de servicios. No en vano, la comunicación deportiva se habría desarrollado en la última década de forma exponencial (5), para satisfacer las demandas de un deporte convertido en uno de los sectores con más potencial económico a nivel mundial (1).

Como hipótesis inicial se partió de que la comunicación desempeña actualmente un papel fundamental de la gestión de las organizaciones deportivas. El principal objetivo consistió en precisar un estado de la cuestión actualizado sobre la importancia del proceso comunicativo dentro de la Gestión Deportiva, fijándose como objetivo complementario describir un plan integral de la Comunicación Deportiva.

\section{MATERIAL Y MÉTODOS}

Se llevó a cabo una revisión sistemática de la bibliografía estructurada en cuatro fases (6): búsqueda, evaluación, análisis y síntesis. Como criterios de inclusión se fijó que se tratara de textos publicados entre 
los años 2000 y 2021 en el contexto hispanohablante, referenciados en las bases de datos Scopus o Dialnet, que incluyeran en su título una combinación de los siguientes términos: "comunicación" o "plan de comunicación" y "deporte" o "gestión deportiva”, y que pertenecieran a las áreas de la Comunicación y la Gestión Deportiva.

De este modo se localizaron inicialmente 2.590 documentos, descartándose duplicaciones, así como todos aquellos documentos referentes a la comunicación aplicada al deporte en campos ajenos a la gestión de la organización deportiva, como didáctica, psicología, entrenamiento deportivo, periodismo deportivo, praxémica, etc. Finalmente se analizó una muestra de 46 publicaciones que abordaban totalmente o en parte el papel de la comunicación dentro de la gestión deportiva. Estas fuentes fueron artículos de revista (30), tesis (3), comunicaciones a congresos (3), monografías y libros (10), que utilizaron metodologías científicas como la encuesta, entrevistas, análisis de contenido, observación participante y revisiones de literatura. Sus fechas de publicación fueron: 13 entre 2000 y 2009, 19 entre 2011 y 2019 y 14 entre 2020 y 2021.

Búsqueda en la literatura

Bases de datos: Dialnet, Scopus

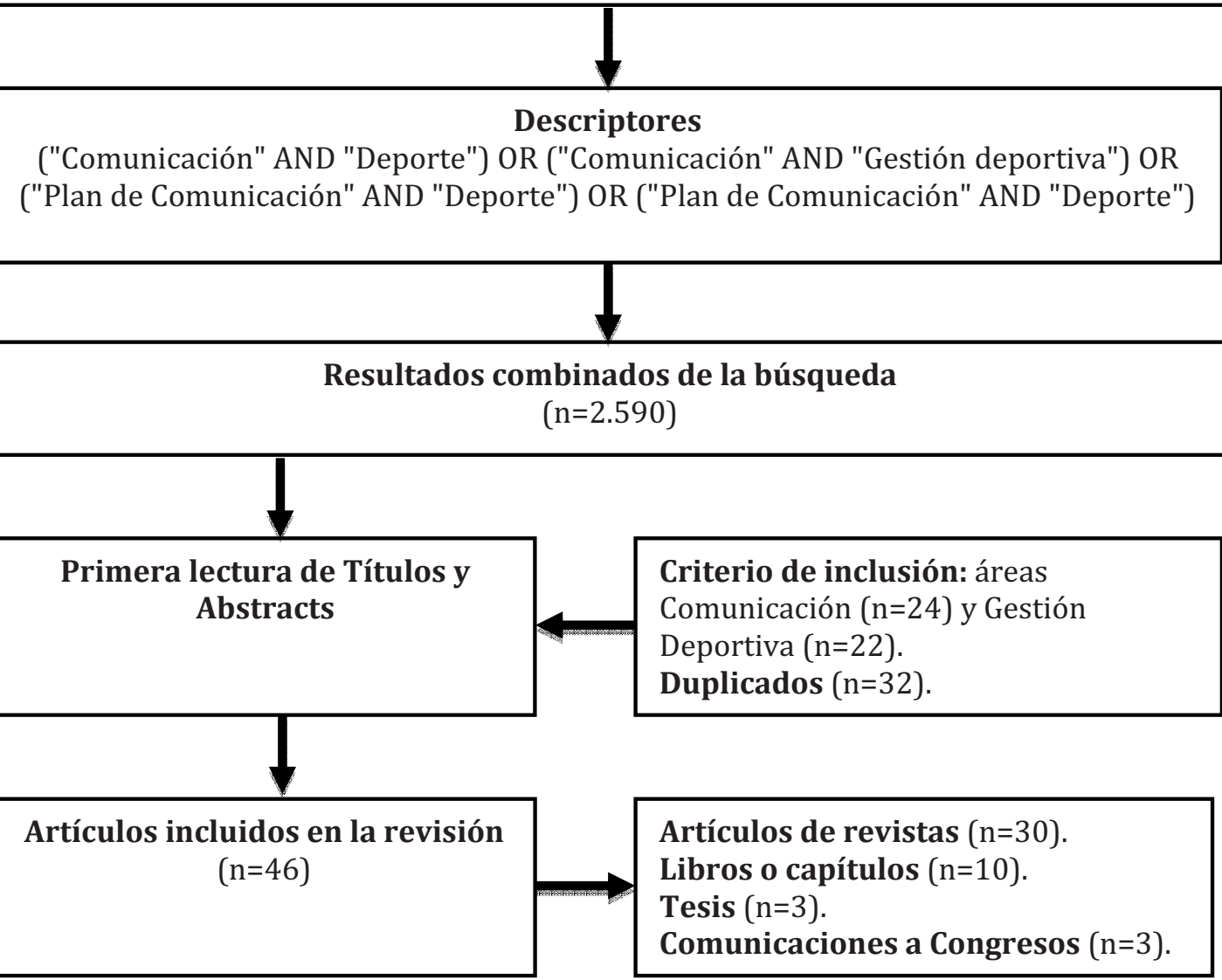




\section{RESULTADOS}

El carácter esencial de la comunicación en tanto herramienta para alcanzar los objetivos de las entidades deportivas ha sido descrito por varios autores $(7,8,9)$. En este sentido, el primer objetivo comunicativo de una entidad deportiva será conseguir ingresos, así como conseguir movilizar y fidelizar a sus públicos (10).

La comunicación deportiva tiene una gran repercusión en la sociedad y la economía actual (10-12). La información deportiva "dispara y consolida las audiencias en televisión (...) tanto en cadenas públicas como privadas, convencionales y digitales, en las que se emiten en abierto y en las codificadas" (13).

Si ya la televisión supuso un notable cambio en la relación entre comunicación y deporte $(12,14)$, internet ha conseguido establecer una interacción de doble sentido, de modo que la comunicación influye sobre el deporte, pero también el deporte promueve cambios en las nuevas tecnologías y formatos de la comunicación. Así, "la confluencia entre la digitalización de los medios audiovisuales, las nuevas redes de comunicación (satélites, fibra óptica) y la globalización de finales del siglo XX" significó la generación de un nuevo "complejo deportivo global", compuesto por el deporte, los medios, la publicidad, el patrocinio y el marketing (14).

Las entidades deportivas han estado en primera línea en la explotación comunicativa de las nuevas tecnologías digitales (15), con la utilización de sitios web, redes sociales y telefonía móvil para “la gestión y el diálogo social con los asociados, generando nuevas herramientas de marketing y comunicación" (14, p. 415), consiguiendo ofrecer a sus aficionados numerosos servicios, desde información de actualidad (resultados, información en directo, crónicas de partidos) a videojuegos, compra de entradas o monografías de jugadores.

A esta lista se une el uso del big data, como factor tecnológico que ha producido cambios profundos en las instituciones deportivas incrementando la productividad, competitividad, internacionalización y la relación entre directivos y colaboradores (16). El big data es considerado por los gestores deportivos como una de las herramientas más importantes, por lo que estiman que su desarrollo "debe ser la absoluta prioridad en la evolución de los métodos de gestión en el área deportiva" (17, p. 141).

Los departamentos de Comunicación de las organizaciones deportivas deben tener en cuenta que internet ha cambiado el modo en que las audiencias asimilan la información deportiva (18). La importancia económica de las redes sociales de los deportistas es tal que la FIFA obliga 
a los futbolistas a un "apagón" durante la disputa del Mundial de fútbol para evitar la concurrencia entre patrocinadores de los deportistas y de la competición (19).

Los deportistas pueden beneficiarse de publicar en las redes sociales mensajes orientados a los aficionados y fotografías de estos, así como informaciones con contenido social para gestionar la reputación y marca personal del deportista (20). La comunicación estratégica no solo proveería valor social, sino que mejoraría la imagen y marca de los deportistas profesionales (21). También se ha recomendado utilizar las redes sociales para publicitar los productos de merchandising, que puede traducirse en ventas.

Además, la transparencia puede dar a las organizaciones deportivas un mejor desempeño en finanzas, marketing y recursos humanos (22). Por ello, estos autores proponen un cuadro de mando integral con toda la información de la organización deportiva para mejorar no solo la gestión interna, sino también la apertura a la sociedad, a través de una política de datos abiertos.

Sin embargo, se ha destacado el escaso aprovechamiento por parte de las organizaciones deportivas de las posibilidades de los medios digitales y la limitada planificación estratégica de la comunicación (23). Pese a los déficits comunicativos de los clubes de fútbol señalados por varias investigaciones $(18,24,25)$, el auge de las redes sociales ha generado un nuevo escenario comunicativo en el que dialogan clubes, futbolistas y seguidores (26).

\section{El proceso de la comunicación en la gestión deportiva}

La comunicación permite a la entidad deportiva relacionarse con sus públicos. Dentro del esquema clásico del proceso comunicativo corresponde a la organización deportiva el rol de emisor que gestiona la comunicación deportiva para alcanzar sus objetivos. Así como el de agente receptor de las informaciones provenientes de sus públicos objetivo.

Partiendo del modelo de Laswell (1948) encontramos cómo agentes emisores en el proceso de la Comunicación Deportiva a los clubes, federaciones y deportistas. Pero también a toda una serie de entidades públicas (servicios deportivos de Ayuntamientos, Diputaciones, Comunidades Autonómas, Universidades) y privadas (empresas de servicios deportivos, cronometraje, gimnasios, organización de eventos, etc.).

Estos emisores tratan de difundir toda una variedad de mensajes como información, publicidad, patrocinio, promoción y relaciones públicas, 
utilizando para ello todos los canales a su disposición, desde lo más clásicos (prensa, radio, televisión) a la amplia variedad de formatos y aplicaciones digitales.

De este modo, se trata de alcanzar a los receptores o públicos objetivo. Por un lado, el cliente externo de la entidad deportiva, compuesto no solo por aficionados, sino también por diferenciados estratos de población en función del carácter de la organización, como aficionados, deportistas, niños, jóvenes, mujeres o tercera edad. No en vano, el éxito en la gestión de un evento deportivo requiere conocer la opinión de los participantes (27). Por otro, se ha descrito la necesidad de tener en cuenta al cliente interno, formado por accionistas, directivos, trabajadores, jugadores y cuerpo técnico. Así, se ha resaltado la importancia del diálogo entre el cliente-interno y las organizaciones en la gestión de la calidad de las organizaciones deportivas (28). Los objetivos de todo este proceso varían desde la obtención de información relevante a aumentar la práctica deportiva, vender productos y servicios, o mejorar la imagen. Por tanto, una planificación integral de la comunicación deportiva pasa por cuidar tanto la comunicación externa como la interna. Precisamente, se ha descrito como uno de los principales retos el ser capaces de realizar un adecuado diagnóstico del entorno interno y externo de las organizaciones deportivas (4).

\section{Comunicación interna}

Frente a la gran atención que se ha prestado a la comunicación externa, se podría haber descuidado la comunicación interna $(29,30)$, en la que la organización trata de involucrar en el proyecto empresarial a todos sus miembros mediante "un proceso comunicacional donde se integran todos los dirigentes de una organización con todos los niveles de trabajadores/as de la misma" (31). La comunicación integra todos los elementos de la organización deportiva, al permitir el flujo de información necesaria para su correcto funcionamiento, a la vez que fortalece valores, reglas y políticas (32).

La comunicación interna utiliza muy diversos instrumentos $(29,33)$ como publicaciones (revista interna, carpeta de bienvenida); tablón de anuncios; notas y reuniones informativas; cursos de formación, actividades sociales; intranet e internet. Además, los servicios deportivos, deben elaborar una memoria anual que incluya apartados como el balance económico, deportivo y social; las actividades deportivas ofertadas y la utilización de las instalaciones. 


\section{Comunicación externa}

La comunicación exterior genera mensajes "dirigidos a crear, mantener o mejorar la relación con los diferentes públicos objetivo del negocio, así como a proyectar una imagen favorable de la compañía o promover actividades, productos y servicios" (34). Los departamentos de Comunicación de las organizaciones deportivas han pasado de encargarse de las relaciones informativas con los medios impresos a una visión más global de la comunicación, “bastante más próximos a las relaciones públicas" (35, p.22).

De tal forma, los departamentos de Comunicación han aumentado tanto sus funciones como su importancia dentro de la organización deportiva $(4,35)$, hasta el punto de que el departamento de Comunicación podría haber sido el que más ha crecido dentro de los clubes de fútbol, por delante de la gestión de las áreas deportiva y financiera $(18,36)$. Ahora se encargan no solo de las relaciones con los medios de comunicación sino también de actualizar las redes sociales, la página web y organizar actos institucionales (25). En cualquier caso, la estructura y funciones de este departamento depende del alcance más local o global que pretenden las organizaciones deportivas (37).

\section{Herramientas para la comunicación externa}

Podemos sintetizar en cinco los instrumentos de comunicación externa (33). En primer lugar, encontraríamos las relaciones con los medios de comunicación, organizadas por una oficina de prensa, que se encarga de difundir sus mensajes mediante notas y ruedas de prensa, así como de la gestión de entrevistas.

La aplicación de las nuevas tecnologías ha mejorado la presencia mediática de los clubes, a través de acciones como las salas virtuales de prensa. Estas páginas con información orientada a los medios de comunicación deben actualizarse constantemente y explotar las potencialidades digitales. Comenzando por las características de multimedialidad, deben aportar no sólo texto, sino también imágenes y vídeos. Además, deben ofrecer "toda la información de la organización de forma simple directa e intuitiva, cumpliendo normas básicas de usabilidad web" (38), entre las que destaca ofrecer un servicio eficiente de búsqueda en el archivo de noticias. Junto a ello, es importante que la sala de prensa virtual permita realizar online consultas y peticiones de información, ya sea mediante formulario o correo electrónico, que sean atendidas y resueltas sin retrasos.

Las entidades deportivas también han creado sus propios medios de comunicación $(18,39)$ para controlar por sí mismos el flujo de la 
información que producen, alcanzando directamente a sus públicos sin la clásica intermediación de los medios (15).

En segundo lugar, encontramos el catálogo de publicaciones de la organización, que incluyen desde formatos tradicionales (revista, folletos, memoria de gestión, manual de identidad corporativa) a toda la variedad de posibles acciones digitales (página web, redes sociales, apps, etc.).

En tercer lugar, la comunicación externa sigue encontrando una importante herramienta en las relaciones públicas, a menudo en forma de entrega de premios, organización de seminarios y congresos; organización de visitas a las instalaciones del club de colectivos interesados; etc.

Para gestionar la publicidad deben tenerse en cuenta todos los canales en función de los recursos económicos disponibles, como son la publicidad directa (correo, teléfono, correo electrónico, internet) y la publicidad exterior. Finalmente, la promoción informa al consumidor del producto, precio, lugar y momento donde lo puede adquirir (40).

Además, las empresas que ofrecen servicios deportivos demuestran su implicación con la transparencia y la calidad en las cartas de servicio, donde se informa a clientes y usuarios sobre las prestaciones de servicios, formas de acceso a estos, horarios y cómo realizar sugerencias y reclamaciones.

La aplicación de estos instrumentos requiere de una actualización constante. Así, por ejemplo, los grandes clubes de fútbol organizan desde hace años visitas a sus estadios e instalaciones deportivas. Bajo diferentes denominaciones (Tour Bernabeu, Territorio Atleti, Barça Stadium Tour) ofrecen conocer la sala de trofeos, pasearse por el estadio, vestuario, banquillos o sala de prensa. Las posibilidades de las nuevas tecnologías han llevado al Barcelona a ofrecer también una visita virtual guiada al Camp Nou de 45 minutos desde dispositivos digitales.

\section{Comunicación corporativa}

Como requisito previo de todo proceso de comunicación se requiere que la audiencia pueda identifi car de un modo claro al sujeto que comunica. Para conseguirlo, la comunicación corporativa permite a la organización deportiva defi nir y transmitir su propia identidad e imagen $(9,31,41)$. La identidad corporativa transmite "lo que la empresa es", mediante una identidad de tipo tanto conceptual (integrada por cuestiones como misión, visión, responsabilidad social) como visual (logotipo, tipografía, colores). 
Un paso inicial en esta definición de la identidad de club deportivo se ejemplifica en casos recientes como la creación del cargo de "director de identidad deportiva" por parte del RCD Espanyol de Barcelona. Se trata de definir tanto cómo quieren jugar como qué valores desean proyectar dentro y fuera del campo. "Precisar un modelo alineado con la historia deportiva del club y que identificara a los más de 400 jugadores de la institución desde la primera plantilla hasta los benjamines" (42).

\section{El patrocinio deportivo}

De igual modo que la organización deportiva busca diferentes canales para sus mensajes, la propia entidad deportiva sirve de canal para otros agentes, bajo el formato de patrocinio. Los patrocinadores consiguen ligar su imagen a los valores deportivos, mientras que la organización deportiva obtiene una valiosa financiación. Así, el deporte profesional "ya no se entiende sin la intervención de las grandes empresas que han ligado su imagen a la de los clubes y deportistas y que, gracias a ello, multiplican sus beneficios en un brevísimo periodo de tiempo". El tipo de patrocinio más habitual es, precisamente, el deportivo, ya que consigue transmitir a las empresas los valores del deporte, como el esfuerzo, la superación de metas o la ejemplaridad (43). Los actos de patrocinio deportivo mueven en torno a los 360 millones de euros anuales según los datos publicados por Infoadex en 2021 (44), constituyendo el 6\% de los medios no convencionales.

Pero no solo debemos pensar en los contratos millonarios de patrocinio del deporte profesional, sino que la multiplicación de actividades más modestas de patrocinio posibilita que miles de clubes deportivos y deportistas participen en competiciones no profesionales. La esponsorización que realizan empresas locales permite a las entidades deportivas renovar equipamiento, y costear gastos de desplazamiento, teléfono, alquileres, etc. Por su parte, las empresas patrocinadoras consiguen que su marca aparezca en noticias de los medios locales, clasificaciones oficiales, fotografías, vídeos, redes sociales, etc.

\section{Comunicación de retorno}

El modelo clásico de comunicación señalaba como colofón la existencia de un flujo de retorno, mediante el cual, el receptor de la información podría convertirse a su vez en emisor. Este proceso de retroalimentación o feedback se ha visto enormemente facilitado por la implantación de los medios digitales.

Sin embargo, la gran potencialidad para generar procesos de interacción entre las organizaciones deportivas y sus seguidores parece 
estar siendo desaprovechado en la práctica. Así, los clubes de fútbol estarían utilizando páginas web y redes sociales sólo como canales de comunicación unidireccional, permitiendo poca interacción con los aficionados (24). Los elementos de interacción-como sorteos, concursos, recibir fotografías de los seguidores o personalizar los mensajes- eran muy poco fomentados por los clubes (25). Según MacLean y Wainwright (2009, cit. en 18) los clubes no estarían aprovechando la interacción que permiten los medios digitales debido a que siguen considerando a los seguidores en tanto consumidores.

Sin embargo, parece firme la apuesta por propiciar la interacción con los aficionados, potenciar los valores de marca y actuar integralmente en medios y soportes (45). La interacción de las organizaciones deportivas con sus públicos a través de las nuevas tecnologías debe planificarse estratégicamente, para cuidar la imagen y los objetivos de éstas (46).

Por tanto, implantar un sistema de comunicación de retorno resulta beneficioso para las entidades. Incluimos aquí las encuestas de satisfacción y los sistemas de quejas, sugerencias y reclamaciones, orientadas hacia los usuarios del servicio deportivo. Todos estos tipos de cuestionarios de calidad percibida buscarían una mayor fidelización de los usuarios y clientes (47). Los cuestionarios online agilizan conocer la opinión de seguidores, deportistas o abonados sobre la calidad y oportunidad del servicio prestado. La creación de estos canales digitales de comunicación de la organización deportiva con sus aficionados y clientes ha sido considerada un deber (48).

\section{La planificación de la comunicación deportiva}

El tamaño de la organización determina el número de profesionales dedicados a la comunicación, desde un único responsable a todo un departamento de comunicación integrado por varios profesionales. Todos los clubes españoles de fútbol de Primera y Segunda División y de baloncesto de la ACB contaban con un departamento de Comunicación, compuesto en un $57 \%$ de casos por uno o dos profesionales, en un $27 \%$ por entre 3 y 5 , y en el $16 \%$ restante por más de 6 personas. Más del $70 \%$ de estos profesionales contaban con una titulación universitaria en alguna rama de las Ciencias de la Comunicación (Periodismo, Publicidad, Comunicación Audiovisual) (49). Además, entre los clubes deportivos que juegan en las máximas categorías de las ligas españolas de fútbol sala, baloncesto, voleibol y balonmano, casi la mitad cuentan con un plan estratégico de comunicación (50). En contraste con estos datos, Monserrat, González y Campillo (46) exponen cómo de las 164 entidades deportivas inscritas en la Federación Española de Vela, sólo el $37 \%$ afirmaba tener un departamento para gestionar la comunicación. 
En el $63 \%$ restante que no contaba con un departamento específico, las funciones de comunicación eran asumidas en su mayoría por empleados de otros departamentos, y en un 10\% por empresas externas.

En el caso concreto de los clubes de fútbol, la segunda década del siglo XXI ha asistido a la profesionalización de los departamentos de comunicación, adaptando sus funciones a las plataformas digitales y desarrollando estrategias de comunicación en una gran variedad de redes sociales $(43,52)$.

Sin embargo, una mayoría de organizaciones deportivas parecen seguir apostando por una planificación informal de su comunicación, fruto de decisiones no programadas. Existe coincidencia en señalar que muy pocas organizaciones deportivas elaboran por escrito un plan de comunicación $(18,47,52,53)$. En este sentido, pese al interés que manifiestan los clubes por las nuevas plataformas de comunicación, la mayoría no tiene bien definidas sus estrategias online (24). Esta forma "improvisada" de actuar podría perjudicar a la imagen y los objetivos de las organizaciones deportivas (23). Aunque no se elabore documentalmente este plan, sí se realizan muchas o parte de las tareas que aparecerían explícitas y sistematizadas en un plan de comunicación.

El plan de comunicación es el documento en el que se describen los objetivos informativos de la organización deportiva y se estructura el proceso de comunicación que se pretende desarrollar. Sintetizando las aportaciones que realizan los autores consultados $(9,29,33,40)$ su elaboración requiere de varias fases. Partiendo del compromiso institucional se pasa a crear el equipo de trabajo que deberá elaborarlo. A continuación, se analizan los entornos externo e interno de la entidad, antes de definir qué acciones concretas de comunicación interna, externa y de retorno se deberán realizar. Para ello se fijará un cronograma de actividades y se estimará con qué presupuesto se cuenta. Tras su ejecución, corresponderá evaluar los resultados alcanzados.

Este proceso de realización del plan de comunicación de la entidad deportiva deberá tener como resultado un documento en el que se detalle cómo se llevará a cabo la comunicación interna y externa, en todos los aspectos descritos anteriormente, desde la relación con los medios de comunicación (oficina, notas y ruedas de prensa, concertación de entrevistas, etc.), a un diseño de los medios propios de comunicación (web, redes sociales, publicaciones, televisión, podcast, etc.), pasando por la generación de un canal de interacción de doble sentido, en el que se recojan y se aprovechen las informaciones procedentes de todos los públicos de la entidad. 


\section{CONCLUSIONES}

El grado de planificación de la comunicación suele presentarse asociado al nivel de éxito y profesionalización de la entidad deportiva, en tanto la planificación integral del proceso de comunicación ayuda a la organización deportiva a alcanzar sus objetivos. Para ello deben tenerse en cuenta tanto los ámbitos interno como externo, y aplicar los instrumentos apropiados en cada caso.

Por un lado, la adecuada atención al cliente interno, sea éste trabajador, directivo o personal deportivo, constituye la base sólida de toda organización deportiva. Un ámbito, el interno, que aparece a menudo olvidado en los planes de comunicación. Sin embargo, la conjunción eficiente de los recursos humanos propios de la organización es un prerrequisito para implantar un sistema óptimo de comunicación externa, donde deberá generarse un flujo bidireccional de la información. La rutina tradicional ha impulsado a las organizaciones deportivas a difundir información sin tener en cuenta previamente las expectativas, necesidades y gustos del público objetivo. Las nuevas herramientas tecnológicas permiten interactuar y obtener de un modo sencillo y en tiempo real una valiosa información de retorno por parte de estas audiencias.

De este modo, el creciente interés por la gestión eficiente de las organizaciones deportivas ha supuesto la implantación de procesos de comunicación cada vez más profesionalizados, indispensables para lograr objetivos tan esenciales como aumentar los ingresos, fidelizar a los usuarios o incrementar la práctica deportiva.

Esta somera descripción resalta el carácter complejo del proceso de comunicación, por lo que optimizarlo requerirá de una gestión planificada llevada a cabo por profesionales especializados en la Comunicación.

\section{DISCUSIÓN}

Disponer de una comunicación eficaz se ha convertido en un factor clave para las organizaciones deportivas en el contexto presente $(7,9,10,29)$, en tanto contribuye decisivamente a lograr los objetivos de gestión.

Instituciones públicas (Ayuntamientos, Diputaciones, Comunidades Autónomas y Estado central) y privadas (clubes, federaciones, comités olímpicos, escuelas deportivas, gimnasios, organizadores de eventos y programas deportivos, gestores de instalaciones, asesores, etc.) se benefician de controlar y dirigir eficientemente los flujos de información dirigidos tanto a su cliente externo como interno. 
Las organizaciones deportivas han centrado sus esfuerzos en la comunicación externa hacia aficionados, abonados, seguidores y público en general, relegando a un segundo plano los procesos de comunicación interna hacia sus propios trabajadores, directivos, socios, etc. $(29,30)$. Sin embargo, canalizar adecuadamente el flujo de comunicación interna es una importante aportación al éxito de la organización, capaz de motivar a los empleados, coordinando los distintos departamentos y agilizando la adaptación a un entorno en cambio continuo.

Por su parte, la comunicación externa debe partir de una definición de la identidad propia de la organización: qué es, cuál es su origen, su historia, su raigambre en su ámbito geográfico de actuación, cuáles son sus objetivos y su misión, a quiénes se dirige, qué es lo que aporta a la sociedad, cómo se plasma esto en una imagen visual, etc. $(9,31,41,42)$. De este modo, se presenta un sujeto cercano y conocido que trata de alcanzar un público objetivo numeroso y heterogéneo, formado tanto por personas físicas (desde aficionados y seguidores a usuarios de servicios deportivos o a diferentes perfiles poblacionales con distintas necesidades de actividad física), como por personas jurídicas, instituciones públicas o empresas privadas, cuyo concurso en diferentes ámbitos (patrocinio, colaboración, trámites, etc.) requiere la entidad deportiva.

Además, las nuevas tecnologías han renovado la consideración de la organización deportiva no sólo en tanto agente emisor de información, sino también como agente receptor. La interacción con sus públicos ha sido tradicionalmente relegada a un papel accesorio, cuando no directamente olvidada. Sin embargo, las herramientas digitales se caracterizan precisamente por su enorme potencial de interacción, que permite fidelizar a los públicos y conseguir un compromiso de los seguidores con la organización deportiva $(18,24,25,45,47)$, ya que la interactividad que promueven los nuevos medios puede fomentar la adhesión a los clubes deportivos (1).

Esta comunicación de retorno se ha establecido mediante canales tan utilizados como las redes sociales, donde se ha vuelto cotidiana la medición de la implicación o engagement de los usuarios (15), en ocasiones con sencillas métricas derivadas del número de likes, compartidos o comentarios que reciben los mensajes en estas redes. La facilidad e inmediatez de este tipo de mediciones contrastan con los recursos tradicionalmente utilizados para obtener información de retorno. Entre ellos se han empleado tanto fuentes externas (observación, asesoría de expertos, encuestas, estudios de mercado, etc.) como internas (encuestas, quejas, sugerencias, resultados económicos, evolución de la clientela, cuantificación del uso de instalaciones, etc.). 


\section{REFERENCIAS BIBLIOGRÁFICAS}

1. Llopis-Goig R. Deporte, medios de comunicación y sociedad. RICYDE Rev Int Ciencias del Deport [Internet]. 2016 Apr 1;12(44):86-9. Disponible en: http: / / www.cafyd.com/REVISTA/044ed.pdf<

2. Madella A, Bayle E, Tome J. The organisational performance of national swimming federations in Mediterranean countries: A comparative approach. Eur J Sport Sci [Internet]. 2005;5(4):207-20. https://doi. org/10.1080/17461390500344644

3. Winand M, Zintz T, Bayle E, Robinson L. Organizational performance of Olympic sport governing bodies: Dealing with measurement and priorities. Manag Leis [Internet]. 2010 Oct 11;15(4):279-307. Available from: http: // dspace.stir.ac.uk/handle/1893/2877

4. Cardona Mejía, L., Cardona Ramírez, S. Tendencias y retos en la gestión de organizaciones deportivas; Perspectivas en Colombia. Sport TK-Revista EuroAmericana de Ciencias del Deporte. 2021;10(1), 59-66. https://doi. org/10.6018/sportk.461671

5. Lobillo G. Estudio sobre la comunicación deportiva. En: Herrero Gutiérrez F, Mateos Martín C, Toledano Buendía S, Ardèvol Abreu A, Trenta M, editores. Del verbo al bit [Internet]. Revista Latina de Comunicación Social; 2017. p. 1978-87. Disponible en: http://www.revistalatinacs. org/16SLCS/2017_libro/100_Lobillo.pdf

6. Codina L. Revisiones sistematizadas: Conceptos, fases y bibliografía [Internet]. 2018. Disponible en: https://www.lluiscodina.com/revisionessistematizadas-fundamentos/

7. Mestre JA. Estrategias de gestión deportiva local. Barcelona: INDE; 2004.

8. Gambau V. Deporte y Comunicación. Introducción al libro de actas. En: Investigación Social y Deporte. Gran Canaria: AEISAD; 2006. p. 11-2.

9. Zapata GL. Comunicar para ganar en el deporte. En: IV Jornadas Iberoamericanas de dirección y gestión deportiva [Internet]. Medellín: Universidad de Antioquía; 2007. p. 1-21. Disponible en: https://revistas.udea. edu.co/index.php/expomotricidad/article/view/335202

10. Pulgar LD. Hacia nuevas políticas de comunicación deportiva. El Publicista. 2001;(50):16-30.

11. Castañón J. Información periodística especializada y comunicación deportiva. En: Congreso La lengua y los medios de comunicación Oralidad, escritura, imagen. Madrid: Universidad Complutense de Madrid; 1996.

12. Billings A. La comunicación en el deporte. Gerona: Editorial Aresta; 2010.

13. Paniagua P. Información deportiva. Madrid: Fragua; 2003. 
14. Moragas M. Deporte y Comunicación: un siglo de sinergias. En: Diálogos sobre el deporte (1975-2020) [Internet]. Barcelona: INDE. 2020. p. 412-21. Disponible en: http://bitly.ws/e2aZ

15. Ballesteros-Herencia, C.A. La narrativa digital del fútbol. Desintermediación de la agenda a través de las redes sociales de los clubes. Hipertext.net. 2021;(22): 85-96. https://doi.org/10.31009/hipertext.net.2021. i22.08

16. Rodríguez Y. La comunicación organizacional en los procesos de dirección de la cultura física y el deporte. Athlos Rev Int ciencias Soc la Act física, el juego y el Deport [Internet]. 2021;(23):78-92. Disponible en: https://dialnet.unirioja.es/servlet/articulo?codigo=7840755

17. Gallardo L, García-Unanue J. Los datos, las analíticas y la digitalización como claves del futuro en la Gestión Deportiva pública y privada. Comun y Hombre [Internet]. 2020 ;(16):133-49. Disponible en: https:// comunicacionyhombre.com/article/los-datos-las-analiticas-la-digitalizacion-claves-del-futuro-la-gestion-deportiva-publica-privada/

18. Olabe-Sánchez F. El gabinete de Comunicación como impulsor de la gestión comunicativa de los clubes de fútbol. Rev Mediterránea Comun [Internet]. 2015;6(1):83-104. Disponible en: http://rua.ua.es/dspace/ handle/10045/44252

19. Suárez O. Deporte y comunicación: dependencia necesaria, dependencia perversa. En: Diálogos sobre el deporte (1975-2020) [Internet]. Barcelona: INDE; 2020. p. 422-7. Disponible en: http://bitly.ws/e2aZ

20. Lobillo G. Estudio de la presencia digital en MotoGP: Estudio de caso Jorge Lorenzo en Instagram. Ámbitos Rev Int Comun [Internet]. 2020; (48):102-22. Disponible en: https: / /institucional.us.es/revistas/Ambitos/48/Estudio_de_la_presencia_digital_en_MotoGP-_Estudio_de_caso_ Jorge_Lorenzo_en_Instagram.pdf

21. Abuín-Penas J., Babiak K. , Martínez-Patiño M.J. Athlete’s philanthropy and social responsibility communication on social media during COVID-19. Journal of Human Sport and Exercise [Internet]. 2020, in press. https: / / doi.org/10.14198/jhse.2022.171.20

22. Muñoz J, Solanellas F. Marco conceptual para el análisis comparativo mediante indicadores de gestión de las organizaciones deportivas. Rev Int Deport Colect [Internet]. 2020;43:87-96. https://doi.org/10.1016/S08404704

23. Monserrat-Gauchi J, González-Redondo P, Campillo-Alhama C. La comunicación organizacional en las Entidades Deportivas Náuticas. Propuesta de modelo estratégico y relacional. Retos [Internet]. 2020;38(38):692-9. https: / / doi.org/10.47197/retos.v38i38.78153

24. Sotelo González J. Deporte y social media: el caso de la Primera División del fútbol español. Hist y Comun Soc. 2012;17:217-30. 
25. Castillo Esparcia A, Fernández Torres MJ, Castillero Ostío E. Fútbol y redes sociales. Análisis de la gestión de Relaciones Públicas 2.0 por los clubes de fútbol. Estud Sobre el Mensaje Periodis. 2016;22(1):239-54.

26. Segarra J, Hidalgo T. Futbolistas en Instagram: análisis del marketing de influencia realizado por los capitanes de Primera División en España. Ámbitos Rev Int Comun [Internet]. 2020;(48):34-55. https://doi. org/10.12795/Ambitos.2020.i48.03

27. Rojo Ramos J, Cerro Herrero D, Madruga Vicente M, Prieto Prieto J. Evaluación de eventos deportivos: el caso del campeonato de España de descenso de cañones 2019. Rev Iberoam Ciencias la Act Física y el Deport [Internet]. 21 de julio de 2021;10(2):60-78. Disponible en: https://revistas.uma.es/index.php/riccafd/article/view/12049

28. Martínez-Moreno A, Díaz, A. Evaluar la gestión de la calidad en los servicios deportivos municipales de la Región de Murcia. Modelo EFQM. J. sport health res. [Internet]. 2017 9(3):301-310. Disponible en: http:// www.journalshr.com/papers/Vol\%209_N\%203/JSHR\%20V09_3_3.pdf

29. Gallardo L, Jiménez A. La Gestión de los Servicios Deportivos Municipales. Vías para la Excelencia. Barcelona: INDE; 2004.

30. Dorado A. La gestión privada en las organizaciones deportivas. En: Lozano J, editor. Futuras claves en la gestión de organizaciones deportivas. Toledo: Universidad de Castilla-La Mancha; 2006. p. 103-16

31. BIC. Cómo elaborar el plan de comunicación. Manuales prácticos de la PYME [Internet]. 2011. Disponible en: http://www.bicgalicia.es/ dotnetbic/Portals/0/banner/ARCHIVOS/Manuales Pymes/ 10_planComunicacion.pdf

32. Giakoni Ramírez F. Competencias en Gestión de los presidentes de Asociaciones Deportivas Chilenas. Pod Sport Leis Tour Rev [Internet]. 2017;6(2):277-93. Disponible en: https://periodicos.uninove.br/podium/ article/view/9540

33. Mediavilla G. La comunicación deportiva: un recorrido hasta la imagen positiva. Gac Gymnos [Internet]. 2001;(9):37-40. Disponible en: https:// dialnet.unirioja.es/servlet/articulo?codigo $=197291$

34. ICEX. Herramientas para la comunicación exterior. El Export [Internet]. 2004;73. Disponible en: https://www.icex.es/icex/GetDocumento?d DocName=DAX2015389913\&site $=$ icexES

35. Lobillo G. Desarrollo comunicacional en los clubes de fútbol españoles. Estudio de caso: Sevilla FC. Rev Iberoam Ciencias la Act Física y el Deport [Internet]. 2018;7(1):34-46. Disponible en: http://www.revistas.uma.es/ index. php/riccafd/article/view/4858

36. Gómez S, Martí C, Opazo M. Características estructurales de las organizaciones deportivas: principales tendencias en el debate académico. IESE Business School. 2008. 
37. Cleland JA. The Changing Organizational Structure of Football Clubs and Their Relationship With the External Media [Internet]. Vol. 2, International Journal of Sport Communication. Loughborough University; 2009. Available from: /articles/journal_contribution/The_changing_organizational_structure_of_football_clubs_and_their_relationship_with_the_external_media/9474917/1

38. Domínguez Quintas S, Álvarez Rodríguez ML, Martí Pellón D. Dirección de comunicación corporativa en internet. Rev Int Relac Públicas [Internet]. 2012;2(3):45-70. Disponible en: http://revistarelacionespublicas. uma.es/index.php/revrrpp/article/view/61

39. Ginesta Portet X. Los medios propios de los clubes de fútbol españoles. De la revista oficial a los canales de TDT. Estud Sobre el Mensaje Periodis [Internet]. 2010;16:145-66. Disponible en: https://revistas.ucm.es/index. $\mathrm{php} / \mathrm{ESMP} /$ article/view/ESMP1010110145A

40. Gutiérrez JF. Fundamentos de Administración Deportiva [Internet]. Medellín: Universidad de Antioquia, Instituto Universitario de Educación Física.; Disponible en: http://viref.udea.edu.co/contenido/menu_alterno/apuntes/ac41-fundamentos.pdf

41. Brotons JM. La comunicación integral aplicada a las entidades deportivas. EFDeportes [Internet]. 2005;89. Disponible en: https://www.efdeportes.com/efd89/comunic.htm

42. Sabatés R. Volver a las raíces para crecer en la élite [Internet]. 2021. Disponible en: https://elpais.com/deportes/2021/05/16/es_laliga/1621117217_405935.html

43. Torres-Romay E, García-Mirón S. Patrocinio deportivo femenino. Situación actual y tendencias. Comun y Género [Internet]. 2020;3(2):125-37. Disponible en: http://dx.doi.org/10.5209/cgen.70373ARTíCULOS

44. InfoaAdex. Estudio InfoAdex de la Inversión Publicitaria en España 2021 [Internet]. 2021. Disponible en: https://www.infoadex.es/home/ wp-content/uploads/2021/02/NP-Estudio-InfoAdex-de-la-Inversión-Publicitaria-en-España-2021.pdf

45. Salinero JC. Nuevas formas de comunicación en el deporte. El twitter de Cristiano Ronaldo. [Internet]. Valladolid: Universidad de Valladolid; 2014. Disponible en: https:/ /uvadoc.uva.es/handle/10324/6252

46. Monserrat-Gauchi J, González-Redondo P, Campillo-Alhama C. La comunicación organizacional en las Entidades Deportivas Náuticas. Propuesta de modelo estratégico y relacional. Retos [Internet]. 2020;38(38):692-9. https://doi.org/10.47197/retos.v38i38.78153

47. Onetti-Onetti W, Castillo-Rodríguez C, Castillo-Rodríguez A. Evaluación de características de las personas mayores y su relación con la calidad percibida de la gestión deportiva. Rev Iberoam Ciencias la Act Física y el Deport [Internet]. 17 de julio de 2018;7(2):110-8. Disponible en: https:// revistas.uma.es/index.php/riccafd/article/view/5095 
48. Budovich, L.S. Business management of the sport industry by considering the digitalization. Journal of Human Sport and Exercise. 2021;16(4proc), S1619-S1624. Https://doi.org/10.14198/jhse.2021.16.Proc4.07

49. Fernández-Souto A.B., Puentes-Rivera I., Vázquez-Gestal M. Gestión de la comunicación en las competiciones deportivas regulares: Fútbol-sala, voleibol, baloncesto y balonmano en España. Retos [Internet]. 2019;36: 9-16. https://doi.org/10.47197/retos.v36i36.66658

50. Sanahuja Peris G. El estado de la comunicación corporativa y el marketing en los clubes deportivos profesionales: retos y modelos para alcanzar la excelencia [Internet]. Castellón de la Plana: Universitat Jaume I; 2012. Disponible en: http://hdl.handle.net/10803/664935

51. Cano Tenorio R, Cristófol Rodríguez FJ, Paniagua FJ. Los clubes de fútbol y sus medios de comunicación propios. Tipos de contenidos más compartidos. En: Romero-Rodríguez LM, Rivera-Rogel D, editores. La comunicación en el escenario digital. Pearson-UTPL; 2019. p. 699-729.

52. Arceo A. El fútbol y las relaciones públicas en España. Chasqui. Rev Latinoam de Comunicación. 2015; 0(84):26-33. Disponible en: https://revistachasqui.org/index.php/chasqui/article/view/1501

53. Cano-Tenorio R, Paniagua FJ. Análisis de la gestión de las redes sociales y salas de prensa online en el club Atlético de Madrid. En: Romero LM, Mañas L, editores. Comunicación institucional en el ecosistema digital: Una visión desde las organizaciones [Internet]. Sevilla: Egregius; 2017. p. 89-112. Disponible en: https://dialnet.unirioja.es/servlet/ articulo? codigo $=7015828$ 